\title{
Transjugular Liver Biopsy Following Orthotopic Whole Liver Transplantation: Challenges and Solutions. A Pictorial Essay
}

\author{
Claire Kaufman ${ }^{1}$ Jordana Gaumond ${ }^{2}$ John Kaufman ${ }^{3}$ \\ ${ }^{1}$ University of California San Diego School of Medicine, San Diego, \\ Address for correspondence Claire Kaufman, MD, University of \\ California, United States \\ 2 The Oregon Clinic, Portland, Oregon, United States \\ 3 Oregon Health Science University Dotter Institute of Interventional \\ California San Diego School of Medicine, 200 W Arbor Drive, San \\ Diego, CA 92103, United States \\ (e-mail: Claire.Kaufman@gmail.com).
}

Radiology, Portland, Oregon, United States

J Clin Interv Radiol ISVIR 2017;1:107-112.
Abstract
Keywords
- Transjugular liver biopsy
- orthotopic liver transplant
- biopsy

Rejection is a common problem after orthotopic liver transplant. The tissue is required for the rapid diagnosis of rejection. Many of these patients have contraindications to percutaneous liver biopsies. Transjugular liver biopsies in patients with liver transplants can be very challenging due to the modified venous anatomy. This article will review the different types of surgical venous anastomosis in patients with whole liver transplants as well as the challenges and techniques to overcome these and successfully perform a transjugular liver biopsy.

\section{Introduction}

In 1964, Dotter first described transjugular liver biopsies (TJLBs) as an experimental procedure. ${ }^{1}$ Since this time, TJLB has proven to be a safe and effective alternative to percutaneous liver biopsy in selected patients. Indications for TJLB include patients with a coagulopathy, thrombocytopenia, ascites, or a need for pressure measurements. ${ }^{2-4}$ The preferred approach for TJLB is via access from the right internal jugular vein (IJV), although the left IJV can be used as well. ${ }^{2,5}$ The literature reports a success rate for right IJV TJLB of $98.8 \%$ with a complication rate of $2.5 \%{ }^{6}$ An additional benefit of TJLB over percutaneous liver biopsy is the ability to measure the hepatic venous and portal pressure gradients, which is a useful predictor of mortality in patients with chronic liver disease. $^{7}$

Rejection is a common problem after orthotopic liver transplant for which tissue diagnosis is crucial. Approximately $60 \%$ of patients have acute rejection, requiring therapy, while $5 \%$ may have chronic rejection. ${ }^{8,9}$ This patient population often has contraindications to percutaneous biopsies such as thrombocytopenia or uncorrectable coagulopathy. In addition, the liver tissue is very fragile posttransplant due to rapid regeneration, leading to the preference to perform TJLB in the early postoperative phase to reduce the risk of hemorrhage. ${ }^{10}$ However, TJLB can be a difficult procedure in this patient population due to the postsurgical inferior vena cava (IVC) and hepatic vein anatomy. ${ }^{3}$ The technical difficulty of the procedure varies with the type of IVC and hepatic venous anastomoses (see - Fig. $\mathbf{1}$ for normal anatomy). There are three common types of anastomoses: Type 1, the end-to-end vena cava anastomoses; type 2, the "piggyback" anastomoses; and type 3; the side-to-side IVC anastomoses. ${ }^{11-13}$

In type 1 anastomosis, the recipient IVC is anastomosed end-to-end with the donor supra- and infrahepatic IVC. In these patients, the resultant IVC and hepatic veins remain in the normal anatomical position ( $\mathbf{- F i g . 2}$ ). The benefit of this type of anastomosis is that it usually closely resembles the native anatomy.

In type 2 "piggyback" anastomosis, the donor IVC is opened suprahepatically and anastomosed to the native hepatic vein orifices, which have also been opened (-Fig. 3). The infrahepatic donor IVC is ligated. This technique is often used in the setting of potential hemodynamic instability as it does not require venovenous bypass and therefore preserves venous return to the right heart during the creation of the hepatic venous anastomosis. ${ }^{14}$ However, 


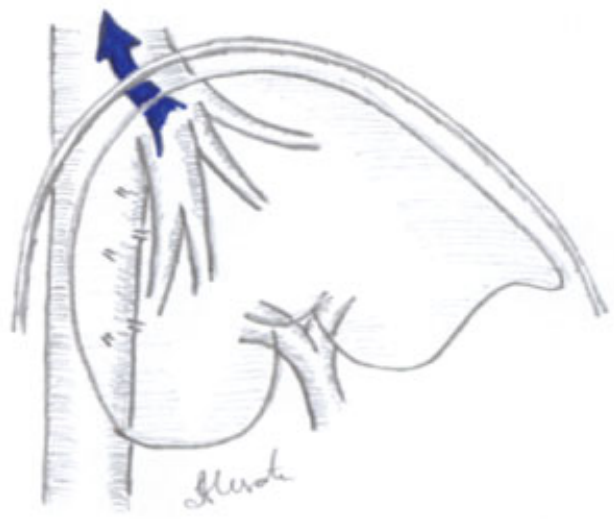

Fig. 1 Normal anatomy of the liver. Normal hepatic vein and IVC relationship is depicted (blue arrow). IVC, inferior vena cava.

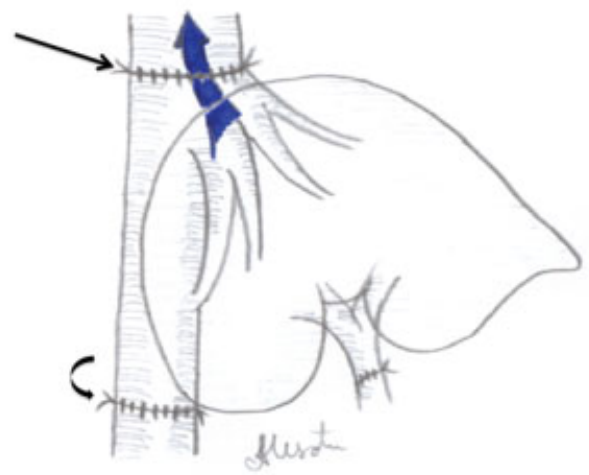

Fig. 2 Type 1 (end-to-end) anastomosis where the recipient IVC is anastomosed in an end-to-end fashion with the donor's supra (arrow) and infrahepatic (curved) IVC. Blue arrow depicts the venous flow of blood from the liver.

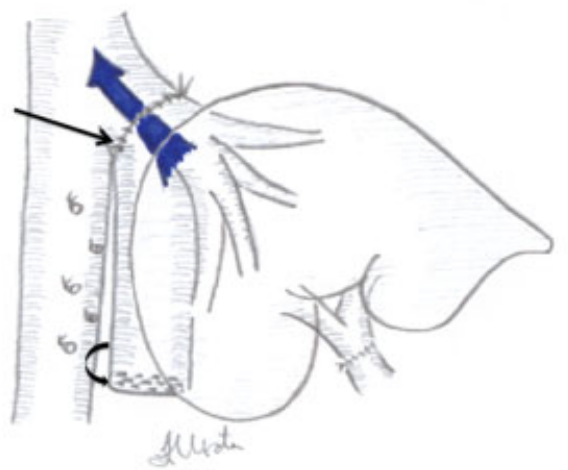

Fig. 3 Type 2 (piggyback) anastomosis where the donor IVC is opened suprahepatically and anastomosed to the native hepatic vein orifices (arrow). The donor IVC infrahepatically is ligated (curved arrow). the type 2 anastomoses typically result in a more acute angulation between the native IVC and donor hepatic veins.

In type 3 side-to-side IVC anastomosis, the donor IVC is ligated suprahepatically and infrahepatically. The posterior segment of the donor IVC is incised longitudinally. The native hepatic veins are ligated at their origins. The donor IVC is anastomosed to the native IVC in a longitudinal side-to-side fashion (-Fig. 4).

\section{Technique}

\section{Preprocedure}

Before TJLB in the patient with a whole liver transplant, the operative report and medical record should be reviewed to determine the type of IVC anastomosis. Next, the available noninvasive imaging should be reviewed for evidence of suprahepatic IVC stenosis, rotation/torsion of the liver, or ascites. A percutaneous biopsy should be considered and therefore the indications for a transvenous biopsy must be reviewed: recent transplant, abnormal and uncorrectable coagulation parameters, low platelet count, the presence of ascites, or the need for hepatic or portal venous pressure measurements.

\section{Transjugular Liver Biopsy}

Venous access using ultrasound guidance preferably from the right IJV should be obtained. A 9 to $10 \mathrm{~F}$ sheath can be placed to minimize trauma due to catheter and device exchanges. Through the sheath, an angled catheter (e.g., Kumpe, Tegt, MPA, and Seeker) can be used to negotiate the suprahepatic IVC and engage a hepatic vein: usually the right hepatic vein. Once successful cannulation of the desired hepatic vein is confirmed, a guidewire is inserted, and over this, the biopsy cannula assembly is advanced into position. If the venous access is via the left jugular vein, a stiff guidewire (Nitrex, Amplatz) should always be used when advancing the biopsy

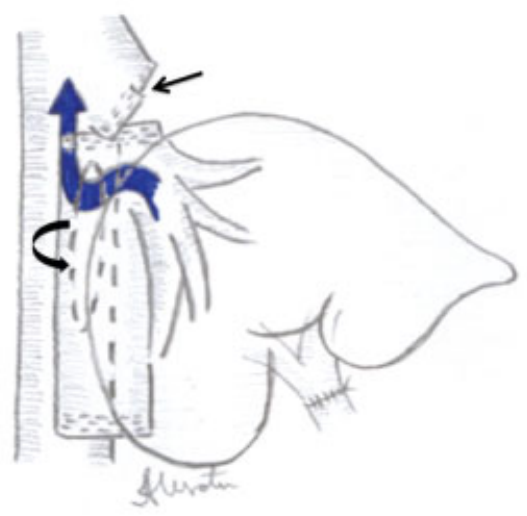

Fig. 4 Type 3 (side-to-side) anastomosis where the donor IVC is ligated supra and infrahepatically. The native hepatic veins are ligated (arrow). The donor IVC is anastomosed to the native IVC through a longitudinal posterior incision in a side-to-side fashion (curved arrow). 
A
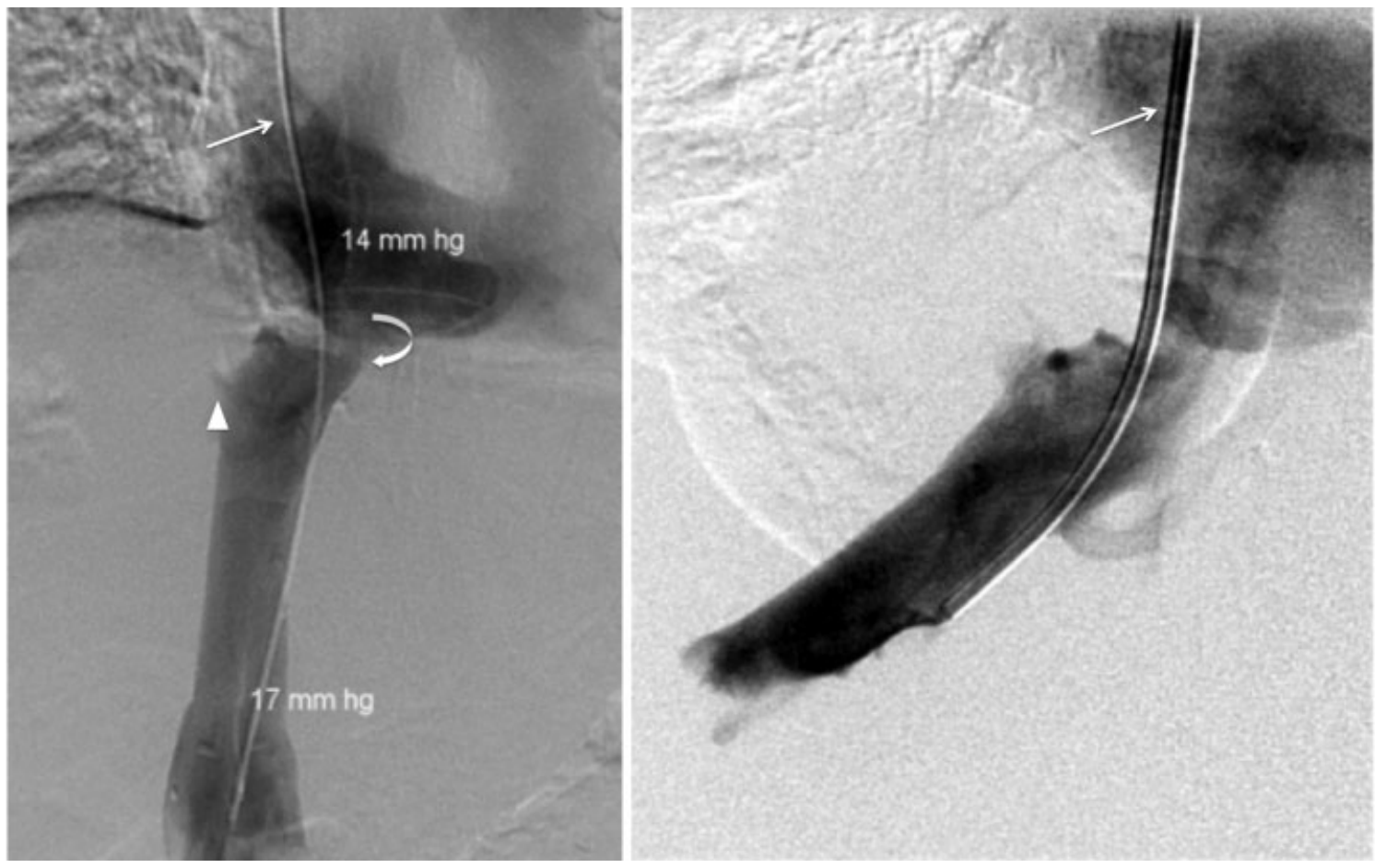

Fig. 5 (A) Venogram performed from a right IJV access (arrow) showing acute angulation at the proximal anastomosis (curved arrow). Hepatic vein orifice is faintly visualized (arrowhead). (B) Successful cannulation of the right hepatic vein when using a left IJV access (arrow). IJV, internal jugular vein.

cannula through the mediastinum. ${ }^{2}$ Before obtaining the biopsy, a contrast injection should be performed to reconfirm the location of the tip of the biopsy cannula assembly. The biopsy needle is then inserted through the cannula. The biopsy needle is then rotated toward the bulk of the hepatic parenchyma, the direction may vary depending upon whether the right, middle, or left hepatic vein has been cannulated, and the biopsy is performed. ${ }^{6}$

\section{Challenge: Acute Angle}

Often for type 1 and type 2 anastomoses, no modifications to the procedure or equipment are required. However, these types of anastomoses are prone to acute angulation of the proximal anastomoses or hepatic vein origin about the suprahepatic IVC. In this case, it may be difficult or impossible to engage the hepatic veins (-Fig. 5). In this situation, there are several technical modifications that may help. A gentle curve may be added to the middle to distal portion of the metal cannula (-Fig. 6). Before introducing this modified system into the patient, the function of the biopsy needle should be tested through the curved cannula. If the access is from the right IJV, as is most commonly performed, the hepatic vein angle with the IVC may be too acute for the stiff metal cannula even with the curved modification. A left internal jugular approach can be considered and may be preferable in this situation as the natural curvature can help direct the cannula into the right hepatic vein (-Fig. 5). An additional option in this circumstance is an intrahepatic transcaval biopsy (-Fig. 7). For this, the biopsy cannula is directed against the wall of the intrahepatic IVC toward the liver.

\section{Challenge: Type 3 Side-to-Side Anastomosis}

Patients with type 3 IVC anastomoses are often the most challenging to biopsy from a transjugular approach (-Fig. 8). The steel cannula must be advanced through the native and then the donor IVC to access a hepatic vein. The hepatic veins

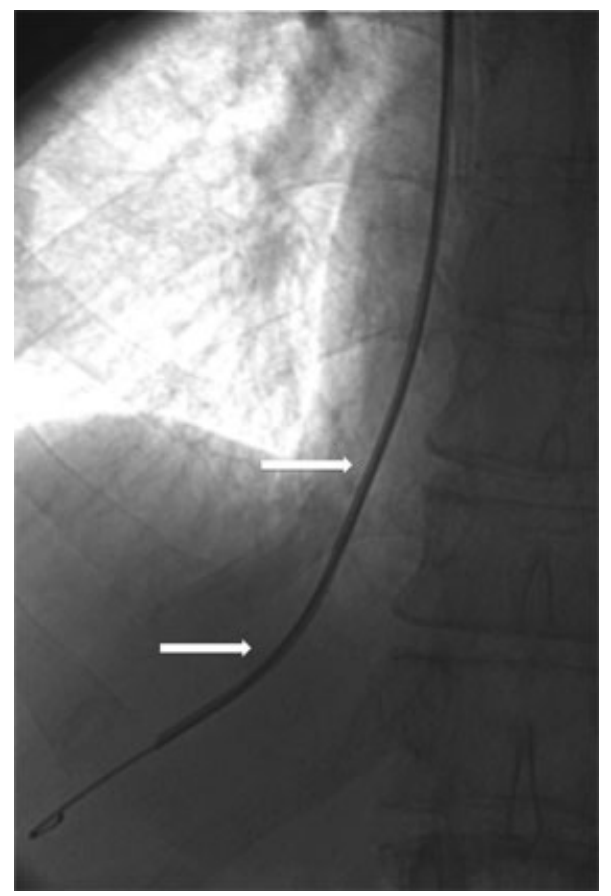

Fig. 6 Fluoroscopic imaging is showing successful cannulation and deployment of the biopsy device in the right hepatic vein after the modification of additional gentle curves (arrows) to the metal cannula. 
A
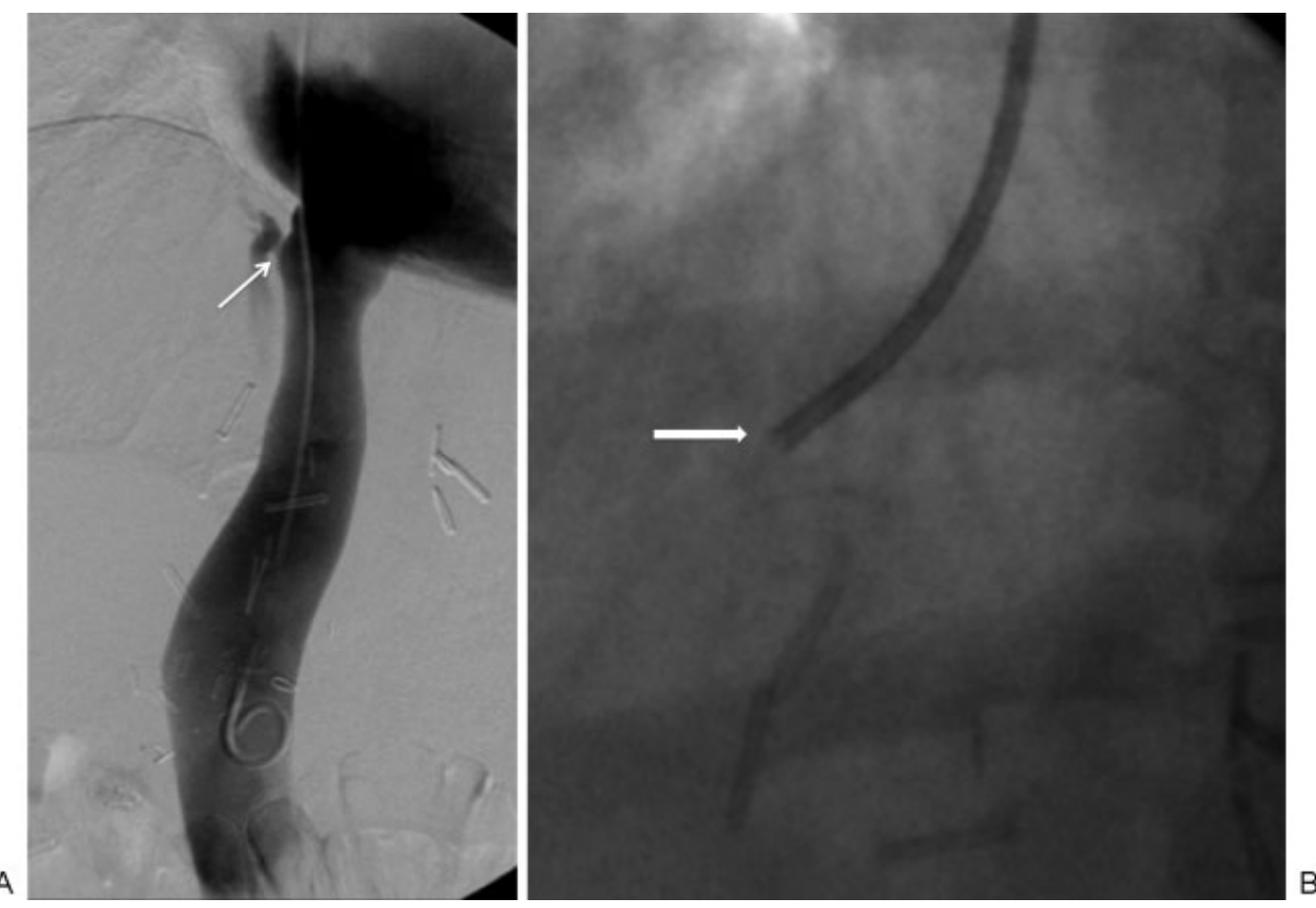

Fig. 7 (A) Cavogram of a patient with a type 1 anastomosis with stenosis of the hepatic vein orifice (arrow). (B) The hepatic veins were unable to be cannulated therefore the biopsy was performed transcaval. Fluoroscopy image is showing the biopsy cannula against the wall of the IVC (arrow).

may be located in the cephalad portion of the donor IVC relative to the side-to-side anastomosis. This orientation is frequently impossible to reach from a jugular approach (-Fig. 8). Several techniques may permit successful biopsy procedure in patients with a type 3 IVC anastomosis. Initially, the donor IVC and hepatic veins should be identified with either an injection of contrast or using intravascular ultrasound (IVUS). As in a traditional TJLB, the hepatic vein should be selected with an angled catheter and a stiff working wire inserted for placement of the biopsy cannula assembly. Similar to any transplant with acute angulation an exaggerated curve can be added to the biopsy cannula to help the tip reach the donor hepatic vein. In addition, if the hepatic vein cannot be successfully catheterized, a transcaval biopsy can also be performed from the donor IVC. Another option in these patients is a transfemoral approach (-Fig. 9). Using the right common femoral venous approach, the donor IVC can be identified using a contrast injection. IVUS guidance if available is recommended and can be inserted from a left femoral vein approach. Often a transcaval biopsy can be safely performed from the donor IVC utilizing IVUS to watch the needle advance into the liver parenchyma.

\section{Challenge: Difficulty with Cannulation}

On occasion, it can be difficult to negotiate from the right atrium into the IVC. If this occurs, the patient should be instructed to hold their breath in deep inspiration while the interventionalist advances the guidewire. Rotating the transjugular biopsy system is also helpful so that the arrow on the cannula points posteriorly. If this fails, it may be of use to oblique the image intensifier into a right anterior oblique view and use an angled catheter to direct the guidewire posteriorly. In addition, another option is to use a torqueable angled hydrophilic wire to obtain hepatic vein or IVC access. At any point during the procedure, it may be useful to obtain

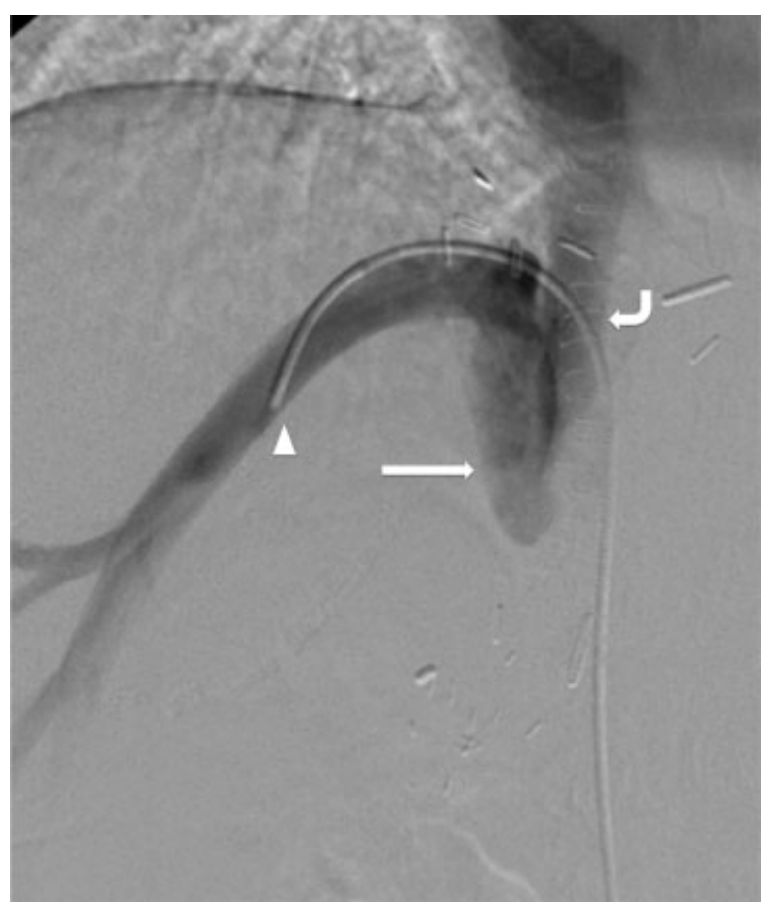

Fig. 8 Venography of the hepatic vein (arrowhead) performed from a femoral vein access in a patient with a type 3 Anastomosis helps delineate the native IVC (curved arrow) and donor IVC (arrow). IVC, inferior vena cava. 

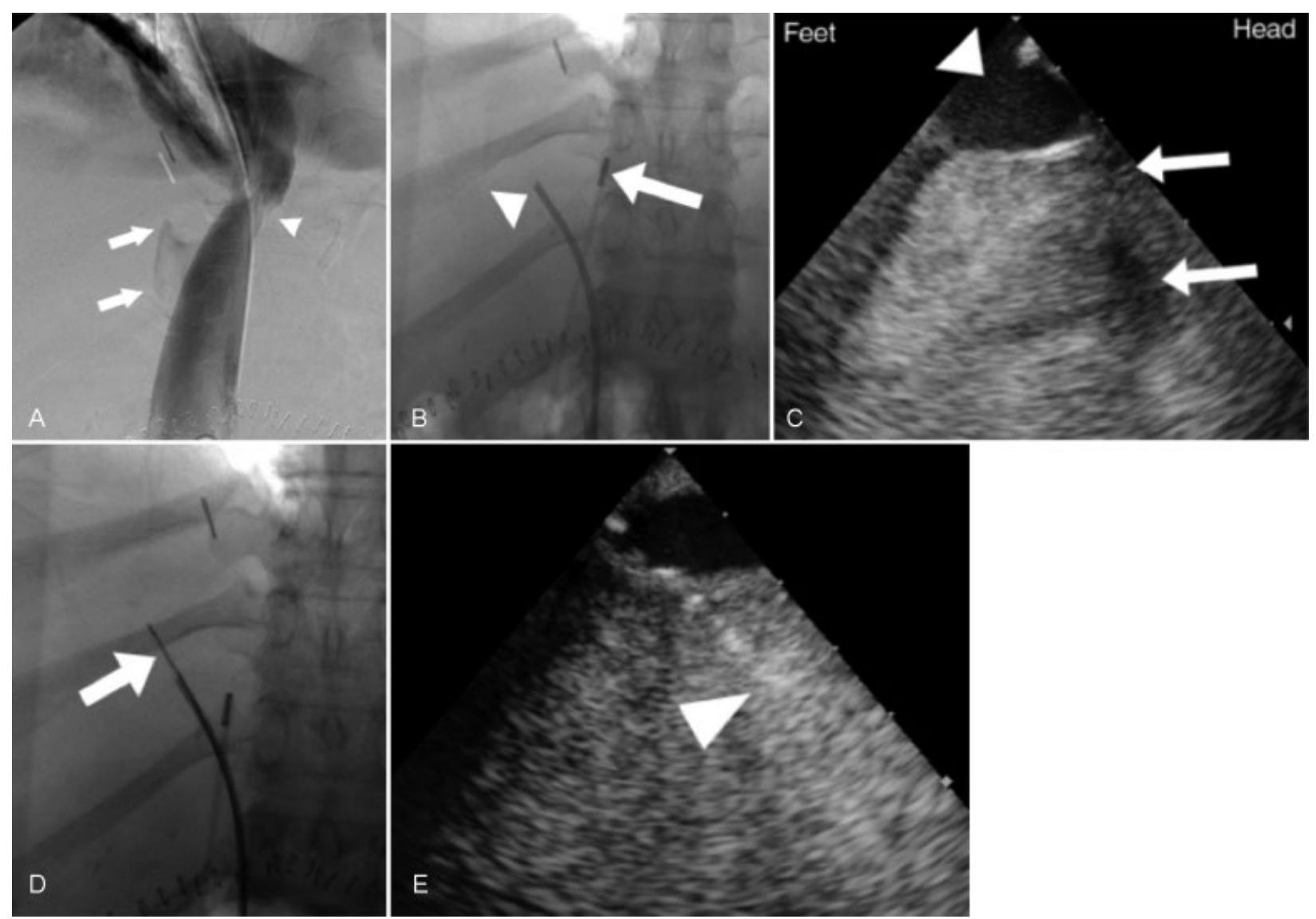

Fig. 9 (A) Cavogram performed from a right internal jugular approach in a patient with a type 3 anastomosis shows a kink in the native IVC (arrowhead) with minimal opacification of the donor IVC (arrows). (B) IVUS probe (arrow) from the left femoral vein is seen in the native IVC, biopsy cannula (arrowhead) from a right common femoral vein approach is seen in the donor IVC. (C) IVUS view from the native IVC (arrowhead) into the donor IVC. The arrows show the right hepatic vein. (D) Biopsy device (arrow) deployed through the donor IVC using IVUS guidance. (E) Corresponding IVUS image of the biopsy needle (arrowhead) in the liver parenchyma. IVC, inferior vena cava; IVUS, intravascular ultrasound.

an IVC venogram from femoral access to delineate the postoperative IVC anatomy. If the issue is difficulty finding the hepatic veins, then an IVC venogram injecting in the intrahepatic IVC can be useful ( - Fig. 7). Another option is to use IVUS assistance (-Fig. 9).

\section{Discussion}

TJLBs are indicated in patients with suspected diffuse hepatic parenchymal abnormalities and abnormal coagulation parameters, ascites, thrombocytopenia, or in whom portal or systemic hemodynamic evaluation is also needed. In addition, posttransplant patients often require a liver biopsy to evaluate for rejection. Frequently these must be done via a transjugular approach for coagulation abnormalities, thrombocytopenia, ascites, or for the concern of bleeding in a patient with recent transplant. ${ }^{10}$ These patients can present technical challenges for transvenous liver biopsy frequently related to the type of IVC anastomoses (-Figs. 2-4). Despite these difficulties, the literature reports a technical success rate in this patient population ranging from 87 to $99.1 \%$ and has found it to be a safe and effective procedure in the posttransplant patient. ${ }^{10,15}$
Patients with type 1 anastomoses typically retain the native anatomic position; however, they can have an angulation of the proximal anastomoses or rotation of the whole liver. These challenges can usually be resolved by gently reshaping the cannula or using a left IJV approach to follow the nature curvatures of the vessels (-Fig. 6). Patients with type 2 anastomoses can be approached similar to those with type 1 anastomoses, but are more likely to require modification of the cannula or a left IJV approach (-Fig. 5).

Patients with type 3 anastomoses are the most challenging and can often not be biopsied from a hepatic vein. A transcaval approach, particularly from the right common femoral vein approach, is often necessary (-Fig. 9). The success of this technique has been published in the nontransplant patient population with a technical success rate of $97 \%$ in a single retrospective review of 66 patients. ${ }^{16}$ In addition, a case series of eight liver transplant patients has been reported in the literature with $100 \%$ technical success; however, none of these patients had whole liver transplants; six modified right lobe, and two dual-left lobe transplants. ${ }^{17}$ Our institution has found this to be of great use in these difficult side-to-side anastomosis cases. 
IVUS can be of great use in any difficult posttransplant TJLB patient. Our experience has found that access via the left common femoral vein is most useful and preserves the right common femoral vein if a transfemoral venous access is needed. The real-time ultrasound can not only delineate the difficult posttransplant anatomy but can also be used during the procedure to confirm appropriate biopsy needle placement within the hepatic parenchyma.

\section{Conclusion}

Orthotopic liver patients frequently require a transvenous biopsy of the liver. This can be successfully performed, however, requires knowledge of the type of transplant venous outflow anastomoses, review of the prior imaging studies, and potential modifications to the biopsy equipment, approach, or imaging guidance.

\section{Acknowledgments}

The authors would specially like to thank Dr. Anatolie Usati for the medical illustrations.

\section{References}

1 Dotter CT. Catheter biopsy. Experimental technique for transvenous liver biopsy. Radiology 1964;82:312-314

2 Yavuz K, Geyik S, Barton RE, et al. Transjugular liver biopsy via the left internal jugular vein. J Vasc Interv Radiol 2007;18(02): 237-241

3 Miller MJ, Smith TP, Kuo PC, Hirasaki KK, Suhocki PV. Transjugular biopsy results in the transplanted liver: comparison of two surgical hepatic venous anastomotic configurations in 269 consecutive biopsies over a 14-year period. J Vasc Interv Radiol 2010;21(04): 508-514

4 Kalambokis G, Manousou P, Vibhakorn S, et al. Transjugular liver biopsy-indications, adequacy, quality of specimens, and complications-a systematic review. J Hepatol 2007;47(02):284-294
5 Gamble P, Colapinto RF, Stronell RD, Colman JC, Blendis L. Transjugular liver biopsy: a review of 461 biopsies. Radiology 1985; 157(03):589-593

6 Mammen T, Keshava SN, Eapen CE, et al. Transjugular liver biopsy: a retrospective analysis of 601 cases. J Vasc Interv Radiol 2008; 19(03):351-358

7 Burroughs AK, Thalheimer U. Hepatic venous pressure gradient in 2010: optimal measurement is key. Hepatology 2010;51(06): 1894-1896

8 Perry I, Neuberger J. Immunosuppression: towards a logical approach in liver transplantation. Clin Exp Immunol 2005; 139(01):2-10

9 Wiesner RH, Demetris AJ, Belle SH, et al. Acute hepatic allograft rejection: incidence, risk factors, and impact on outcome. Hepatology 1998;28(03):638-645

10 Kim KR, Ko GY, Sung KB, et al. Transjugular liver biopsy in patients with living donor liver transplantation: comparison with percutaneous biopsy. Liver Transpl 2008;14(07):971-979

11 Belghiti J, Panis Y, Sauvanet A, Gayet B, Fékété F. A new technique of side to side caval anastomosis during orthotopic hepatic transplantation without inferior vena caval occlusion. Surg Gynecol Obstet 1992;175(03):270-272

12 Cherqui D, Lauzet JY, Rotman N, et al. Orthotopic liver transplantation with preservation of the caval and portal flows. Technique and results in 62 cases. Transplantation 1994; 58(07):793-796

13 Ducerf C, Rode A, Adham M, et al. Hepatic outflow study after piggyback liver transplantation. Surgery 1996;120(03): 484-487

14 Nishida S, Nakamura N, Vaidya A, et al. Piggyback technique in adult orthotopic liver transplantation: an analysis of 1067 liver transplants at a single center. HPB (Oxford) 2006;8(03):182-188

15 Azoulay D, Raccuia JS, Roche B, Reynes M, Bismuth $\mathrm{H}$. The value of early transjugular liver biopsy after liver transplantation. Transplantation 1996;61(03):406-409

16 Cynamon J, Shabrang C, Golowa Y, Daftari A, Herman O, Jagust M. Transfemoral transcaval core-needle liver biopsy: an alternative to transjugular liver biopsy. J Vasc Interv Radiol 2016;27(03): 370-375

17 Li FQ Ko GY, Sung KB, et al. Transfemoral liver biopsy using a Quick-Core biopsy needle system in living donor liver transplantation recipients. Liver Transpl 2014;20(10):1178-1184 